\title{
Synthesis and Characterisation of Silver Nanoparticles in Different Medium
}

\author{
G. Alagumuthu* , R. Kirubha \\ Department of Chemistry Sri Paramakalyani College, Manonmaniam Sundaranar University, Alwarkurichi, India \\ Email: *alagupathi@yahoo.co.in
}

Received April 14, 2012; revised May 30, 2012; accepted June 24, 2012

\begin{abstract}
Silver nanoparticles were synthesized in different alcoholic medium such as ethylene glycol and n-butyl alcohol by solvothermal method. The nanoparticles have been successfully synthesized by reducing silver nitrate with the above solvents in the presence of trioctyl phosphine oxide (TOPO) as the capping agent at room temperature for $1 \mathrm{~h}$. Electron microscopy, X-ray diffraction, and absorption spectra have been used to investigate the products, and the mechanism is proposed to interpret the controlled synthesis of the products. The results indicate that this approach provides a versatile route to prepare silver nanowires and nanoparticles with controllable diameters. The formation of nano products by this method is rapid, simple and stable.
\end{abstract}

Keywords: Silver Nanoparticles; TOPO; Ethylene Glycol; N-Butyl Alcohol

\section{Introduction}

Nanoparticles are being viewed as fundamental building blocks of nanotechnology. The most important and distinct properties is that they exhibit larger surface area to volume ratio. They exhibit completely new or improved properties based on specific characteristics such as size, distribution and morphology [1]. Advances over the past two decades reveals that the silver nanoparticles (NPs) possess unique optical, electrical and catalytic properties [2,3]. During the past few years, the field of silver NPs preparation has witnessed tremendous growth in synthetic sophistication and depth of characterization [4].

Photochemical method of silver nano particles is prepared by using high molecular weight carbohydrate and carbohydrate-based dendrimers as reducing and stabilizing agent $[5,6]$. A number of preparation routes have been reported for the synthesis of silver nanoparticles. For example facile method, thermal decomposition of silver compounds, electrochemical, sonochemical, microwave assisted process and recently via chemical route $[7,8]$. The solvothermal method was also employed to synthesize silver nanoparticle. This technique is based on thermal decomposition of metallic compound in organic solvent and has been successfully applied for the synthesize of various types of nano sized metal oxide with large surface area, high crystallinity and high thermal stability. The influences of reaction conditions, viz., type of solvents, concentration of precursors and reaction tempera-

${ }^{*}$ Corresponding author. ture, on the physical properties of the synthesized nanorods as well as mechanism were studied [9].

Different approaches have been used to synthesize silver nanowires. The template-directed approaches were the most effective and widely used. Macroporous membranes, mesoporous materials, carbon nanotubes, DNA channels, organic nanotube arrays and silica gels have been used as physical templates to guide the growth of nanowires. Though above-mentioned methods can ensure a good control over morphology of final product and allow obtaining metal wires with high aspect ratios, the additional removal of these physical templates may complete the synthetic procedures and limit the scale at which materials can be synthesized [10].

The purpose of this paper is to describe a facile way for synthesis of silver nanowires and particles through the solvothermal method by the reduction of silver nitrate with ethylene glycol and n-butyl alcohol are an appropriate medium in the presence of TOPO as an adsorption agent and characterized herein.

\section{Experimental}

Silver nanoparticles were synthesized by using two different solvents, viz., n-butyl alcohol and ethylene glycol. Trioctyl phosphine oxide (TOPO) was used as the capping agent. For the synthesis of silver nanoparticles, the simple solvothermal method was used at room temperature. All the aqueous solutions were prepared using ultrahigh purity water which was purified by a Milli-Q 
system. In a typical synthesis procedure, $20 \mathrm{ml}$ ethylene glycol was added to $20 \mathrm{ml}$ of $0.5 \mathrm{M}$ TOPO solution and stirred vigorously. This mixture was then injected drop by drop into $20 \mathrm{ml}$ of $0.1 \mathrm{M} \mathrm{AgNO}_{3}$ solution in a beaker and placed in a magnetic stirrer that was operated at room temperature for an hour. The solution turned colloidal and grey in colour, which suggested the formation of silver nanoparticles.

\section{Characterization of Silver Nanoparticles}

A different value will be obtained depending on whether a technique is sensitive to the medium size or the mean size, and whether that median and mean is number weighed or volume weighed. In addition, nanoparticles are not always single crystals. Many techniques viz., XRD, SEM, FT-IR and UV-Vis spectra, yield a crystallite size as well as the size of the aggregate particles. XRD measurements are performed using a Philips diffractometer of ' $X$ ' pert company with nano chromatized $\mathrm{Cu} \mathrm{K} \alpha 1(\lambda=1.54060 \AA)$ radiation, the size is determined from the width of XRD peaks using Scherrer's formula,

$$
D=0.86 \lambda / \beta \cos \theta
$$

where,

$D=$ is the average crystalline size;

$\lambda=$ is the $\mathrm{X}$-ray wave length;

$\beta=$ is the full width and half maximum (FWHM);

$\theta=$ is the diffraction angle.

FT-IR measurements is undertaken in order to confirm the formation of crystalline nanocrystals and identify adsorbed species onto the crystal surface. Generally, FT-
IR is recorded using Nicolet FT-IR spectrometer mode impact 400 . The spectra were recorded at wave number in the range of 400 and $4000 \mathrm{~cm}^{-1}$. The UV-Vis absorbance spectra were recorded using a Shimadzu UV-1800 spectrophotometer (Japan), and $1 \mathrm{~mm}$ path length quartz cuvettes were used for the measurements of visible spectra. The electronic images were made on Hitachi S-4500 SEM Analyzer.

\section{Results and Discussion}

A drop wise addition of ethylene glycol-TOPO and nbutyl alcohol-TOPO to silver nitrate solution resulted in the intermediate change in colour from light brown to grey. The size and structure of the obtained nanoparticles were confirmed by the typical XRD patterns of the products taken with a bigger quantity of the dried sample and shown in Figures $\mathbf{1}$ and 2. The XRD pattern indicates that the presence of three diffraction peaks, which agreed well with (111), (200) and (220) diffractions of face centered cubic silver(JCPDS File No. 04-0783 from ASTM) The diameter of the nanowires is calculated by using Debye-Scherer's formula (11) and it was found as $9.99 \mathrm{~nm}$. The final product is all composed of metallic silver, indicating that high purity of fcc structure with all parameters $a=4.065$. Similarly the XRD patterns of silver nanoparticles synthesized using n-butyl alcohol solvent in Figure 2 shows characteristic peak at $2 \theta=38.5$, marked with (111). It confirms the hypothesis of mono crystallinity. The sharpening of peak clearly indicates that the particles are in nano region. The particle size was

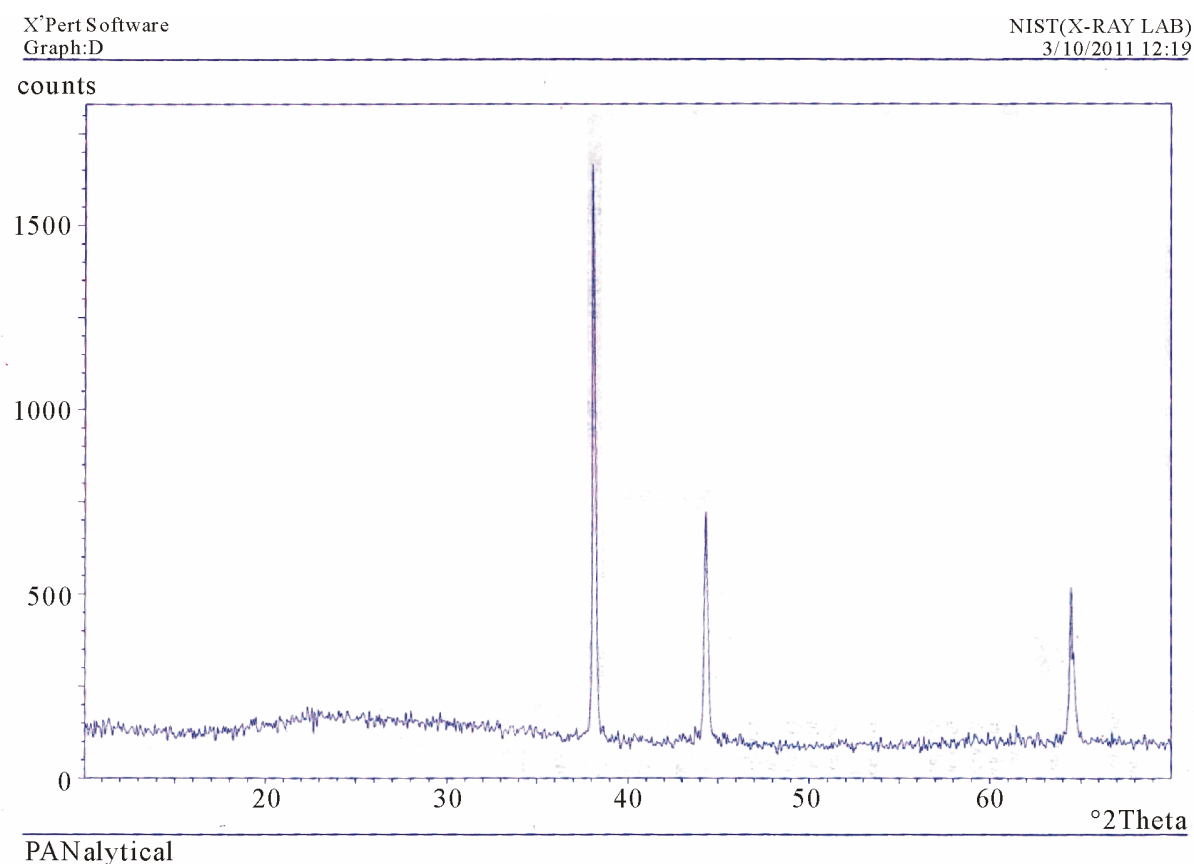

Figure 1. XRD Pattern of pure silver nanoparticles in ethylene glycol. 


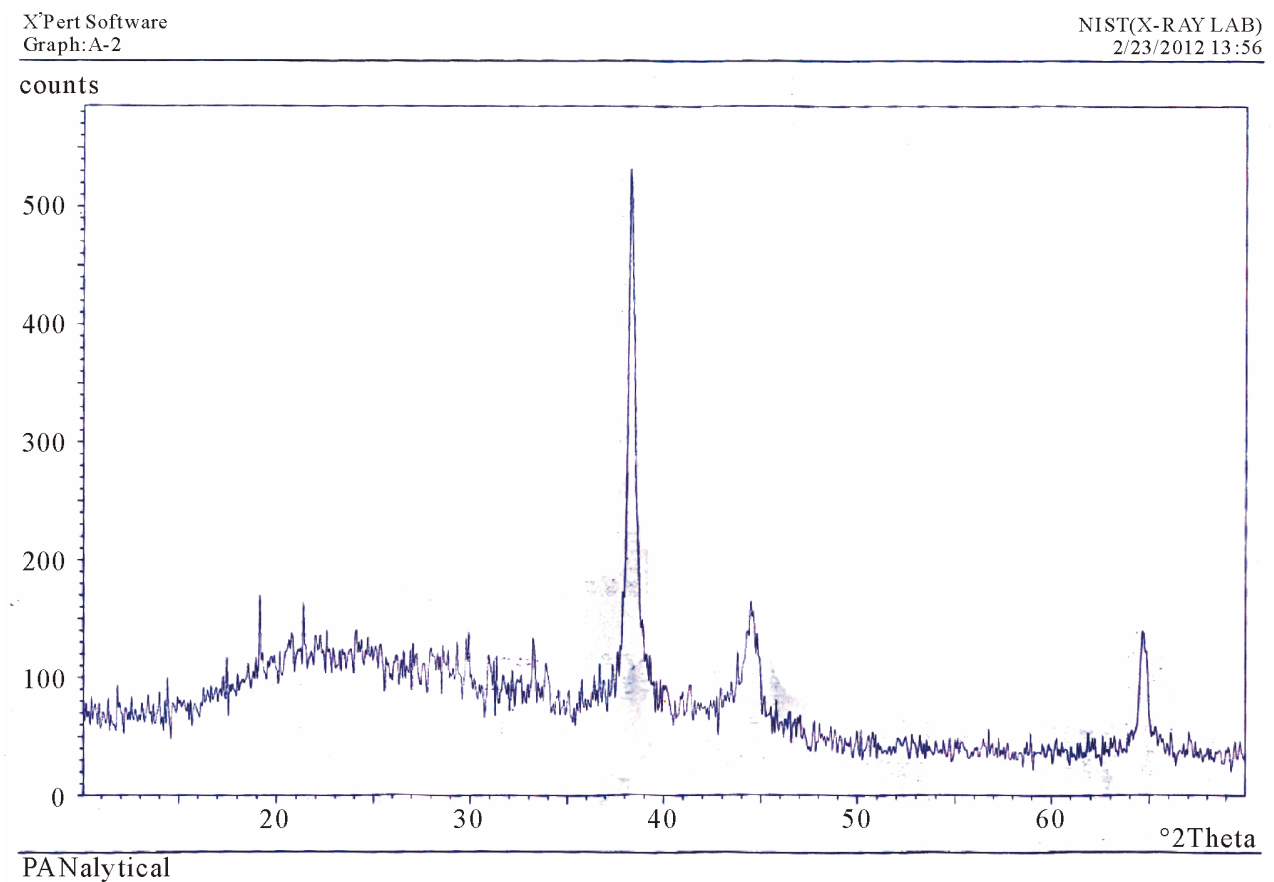

Figure 2. XRD Pattern of silver nanoparticles in n-butyl alcohol.

also found and the value is higher than the ethylene glycol solvent. Hence particle size of silver nanoparticles is $9.99 \mathrm{~nm}$ and $42 \mathrm{~nm}$ for ethylene glycol and n-butyl alcohol respectively. The data are compared with the reported pure silver nitrate sample.

\section{Optical Absorption}

The optical absorption spectra of the nanoparticles were measured using a USB-2000 UV-VIS spectrophotometer. The powder material has been suspended in glycerol using magnetic stirrer and their optical absorption spectra has been recorded at room temperature over the range 300 to $600 \mathrm{~nm}$. It reveals that the absorption edges are blueshifted with decreasing particle size. This is confirmed by quantum confinement effect in the nanoparticles [12].

The fundamental absorption, which corresponds to electron excitation from the valance band to conduction band, can be used to determine the value of the optical band gap energy. The value of optical band gap is calculated by extrapolating the straight line portion of $(\alpha \mathrm{h} v)^{2}$ vs $\mathrm{h} v$ axis [13]. The obtained values are $0.095 \mathrm{eV}$ and $0.046 \mathrm{eV}$ for ethylene glycol and n-butyl alcohol respectively. This fact explains the bigger particle size obtained from a slow reaction, which is associated with a more important ripening contribution to the growth [14]. There- fore the silver nanoparticle has larger band gap energy value.

The interaction of nanoparticles obtained with the different medium was confirmed by FT-IR spectra. This was shown in Figures 3 and $\mathbf{4}$. The peak observed at $3080 \mathrm{~cm}^{-1}$ corresponds to stretching vibration of $-\mathrm{OH}$ bond. After the reduction with the $\mathrm{AgNO}_{3}$, the shift in the peak at $413.21 \mathrm{~cm}^{-1}$ towards lower frequency is attributed to the formation of nanoparticles. The stretching frequency of N-O is observed at above $1385 \mathrm{~cm}^{-1}$. This is confirmed by the changes in the peak shift of silver nitrate and silver nanoparticles appeared in different medium. In this regard, the mechanism of solvothermal process seems to be similar to that of the polyol process demonstrated for the synthesis of highly crystalline nano materials from III group to V group semi conductors and silicon [15].

SEM images of the silver nanoparticles products prepared at ethylene glycol and n-butyl alcohol medium. The image of silver nanoparticles in ethylene glycol medium is shown in Figure 5. The effect of TOPO on the morphology of silver nanoparticles products can be explained by the selective adsorption of capping agent on the surface of the silver nanoparticles. Notably, the TOPO and different solvents play an important role on growth of silver nanoparticles. The silver nanoparticles formed were predominantly cubical with uniform shape. It is known that the shape of metal nanoparticles considerably change their optical and electronic properties. This shows that the formation of silver nano sample in the ethylene glycol medium is significant and reveals that the size of the silver wire is in nano form.

\section{Conclusion}

The present work shows that the silver nano products were synthesized using TOPO-mediated solvothermal 


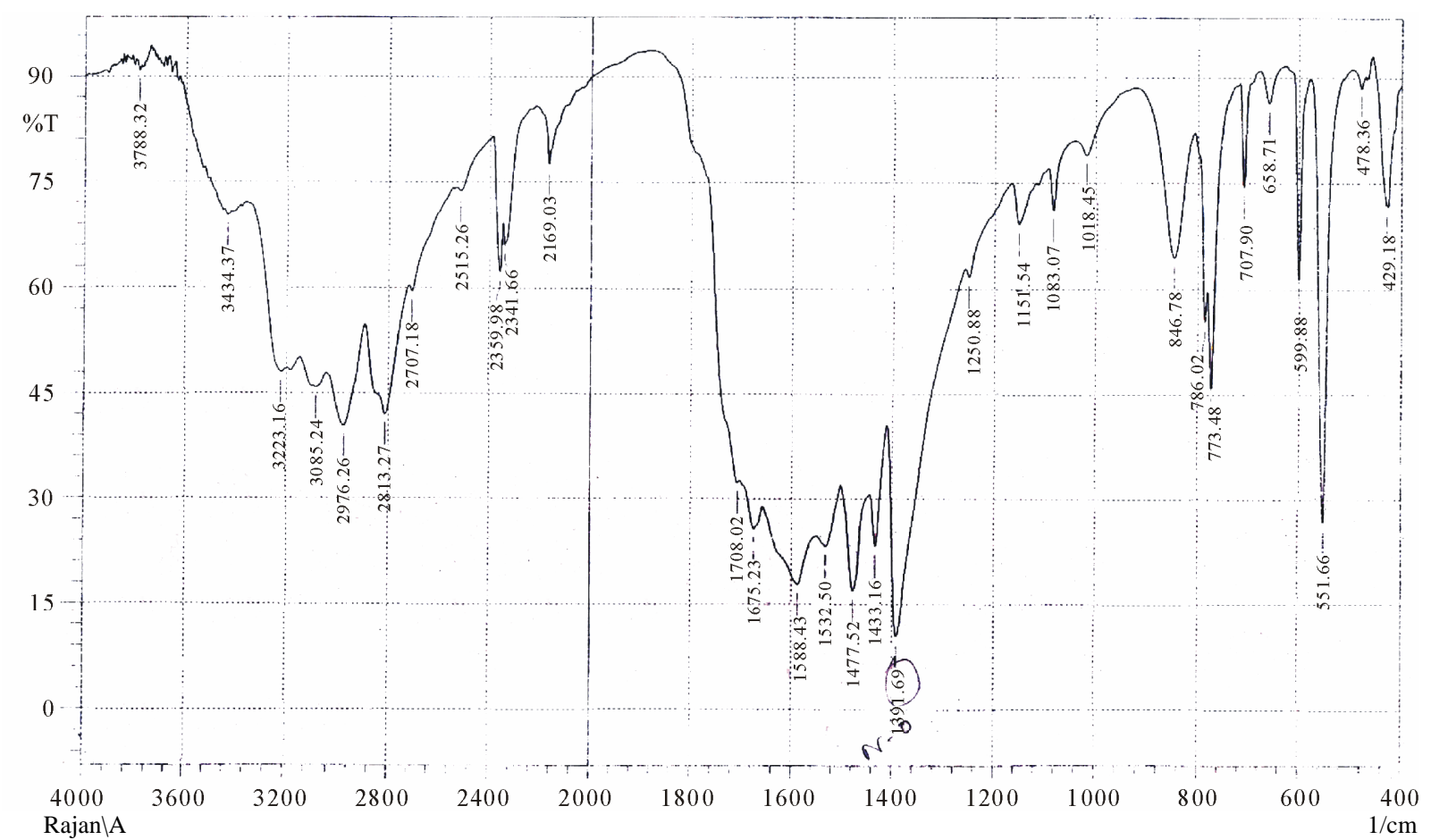

Figure 3. FTIR-Spectra of Silver nanoparticles in ethylene glycol.

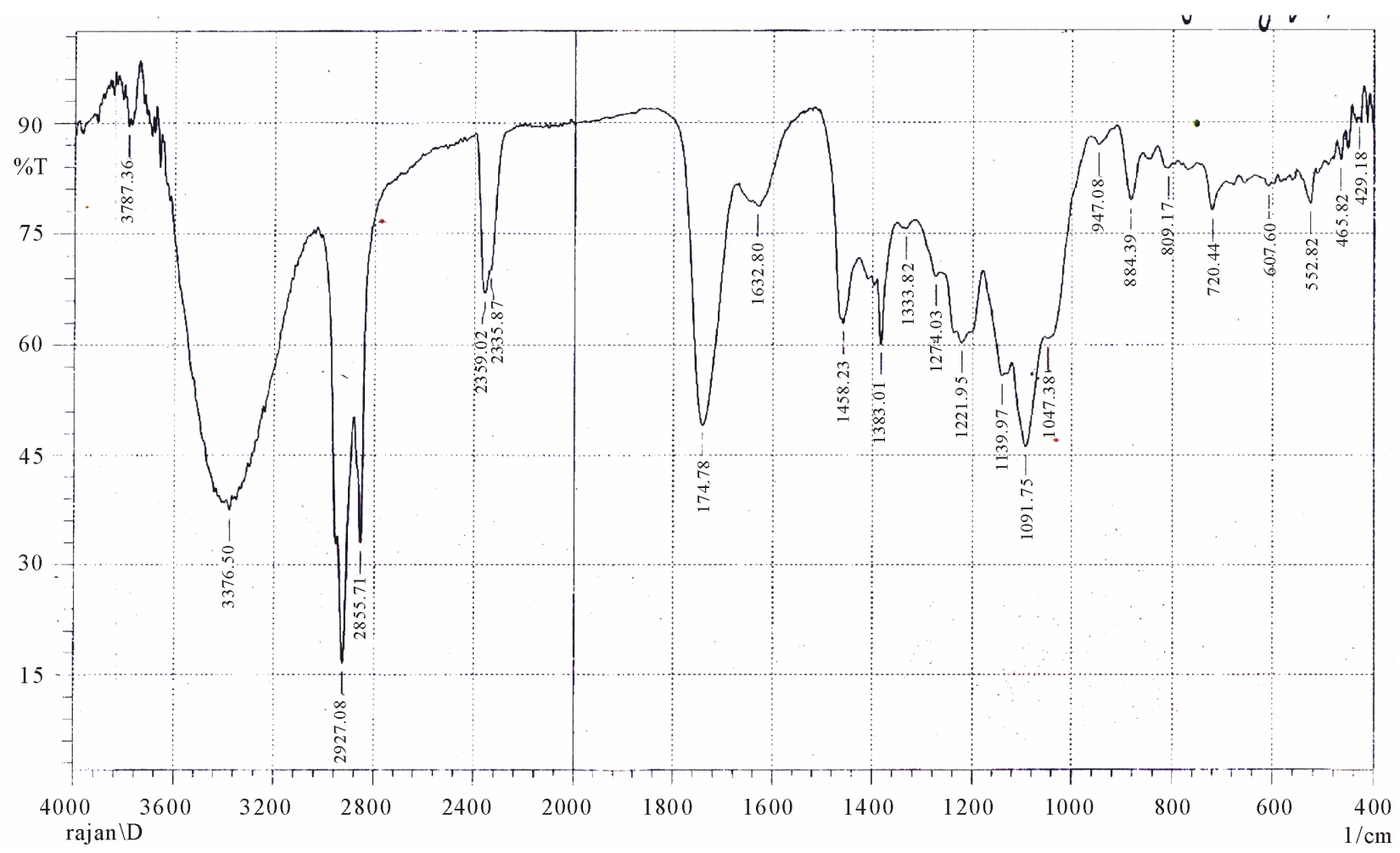

Figure 4. FTIR-Spectra of Silver nanoparticles in n-butyl alcohol.

process. The capping agents have the capability of effective covering and stabilize the newly formed nano compounds. The face centered cubic structure and use of TOPO with proper concentration; both play an important role in confining the growth of silver nanowires to the ID mode. Silver nanowires with a uniform diameter of 9.99 $\mathrm{nm}$ were obtained. The end-to-end assemblies of silver nanowires were formed during the reaction process, and 


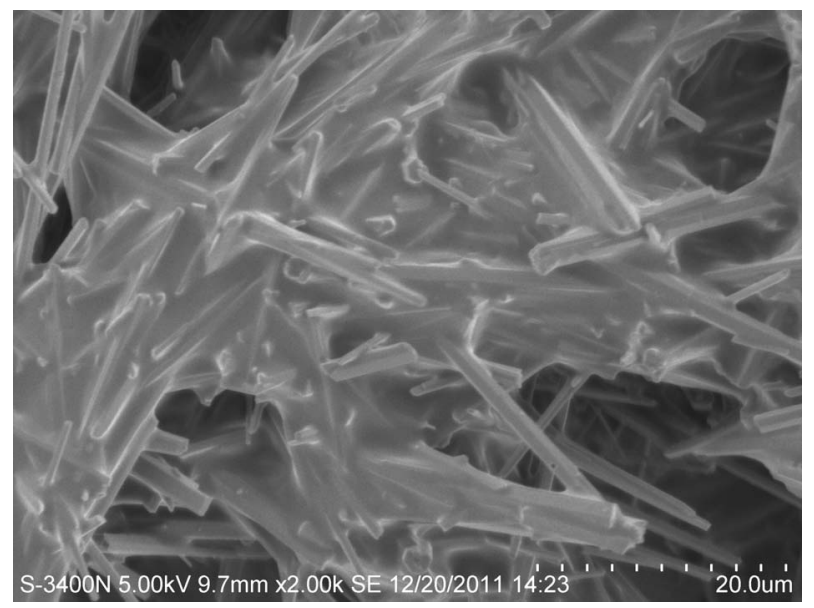

Figure 5. SEM image of Sliver nanoparticles in ethylene glycol.

the obvious spacing between two straight silver nanowires would gradually disappear and probably to be filled with silver atoms. This method can be used to select the appropriate solvent for the preparation of silver nanowires with desired aspect ratio, indicating preferential potential for applications in fabricating future nano electronic devices.

\section{REFERENCES}

[1] D. Jain, H. K. Daima, S. Kechhwaha and S. L. Kothani, "Synthesis of Plant-Mediated Silver Nanoparticle Using Papaya Fruit Extract and Evaluation of Their Antimicrobial Activity,” Materials Science and Applications, Vol. 4, No. 3, 2009, p. 5573.

[2] A. Henglein, "Physicochemical Properties of Small Metal Particles in Solution: 'Microelectrode' Reactions, Chemisorption, Composite Metal Particles, and the Atom-toMetal Transition,” Journal of Physical Chemistry, Vol. 97, No. 21, 1993, pp. 5457-5471. doi:10.1021/j100123a004

[3] Y. A. Krutyakov, A. A. Kudringkii, A. Y. Olenim and G. V. Lisichkui, "Synthesis and Properties of Silver Nanoparticles: Advances and Prospects," Russian Chemical Reviews, Vol. 77, No. 3, 2008, pp. 233-257. doi:10.1070/RC2008v077n03ABEH003751

[4] A. Pal, S. Shah and S. Devi, "Preparation of Silver, Gold and Silver-Gold Bimetallic Nanoparticles in W/O Microemulsion Containing TritonX-100," Colloids and Surfaces: Physicochemical and Engineering Aspects, 302, No.
1-3, 2007, pp. 483-487.

[5] J. Kasthuri, S. Veerapandian and N. Rajendran, "Biological Synthesis of Silver \& Gold Nanoparticles Using Apiin as Reducing Agent," Colloids and Surfaces B: Biointerfaces, Vol. 68, No. 1, 2009, pp. 55-60.

[6] L. Balogh and D. A. Tomalia, "Poly(Amidoamine) Dendrimer-Templated Nanocomposites. 1. Synthesis of Zerovalent Copper Nanoclusters," Journal of the American Chemical Society, Vol. 120, No. 29, 1998, 7355-7356.

[7] K. Patel, S. Kapoor, D. P. Daul and T. Murherjee, Journal of Chemical Sciences, Vol. 117, No. 1, 2005, pp. 5360. doi:10.1007/BF02704361

[8] J. J. Zhu, X. H. Liao, X. W. Zaho and H. Y. Hem, "Preparation of Silver Nanorods by Electrochemical Methods," Materials Letters, Vol. 49, No. 2, 2001, pp. 91-95. doi:10.1016/S0167-577X(00)00349-9

[9] Y, G. Sun, B. Mayers, T. Herricks and Y. N. Xia, "Polyol Synthesis of Uniform Silver Nanowires," Nanoletters, Vol. 3, No. 7, 2003, pp. 955-960.

[10] Y. Xia, P. Yang, Y. Sun and Y. Wu, “One-Dimensional Nanostructures: Synthesis, Characterization, and Applications," Advanced Materials, Vol. 15, No. 5, 2003, pp. 353-389. doi:10.1002/adma.200390087

[11] C. Chem, B. Yu, J. Liu, Q. Dai and Y. Zhu, "Investigation of $\mathrm{ZnO}$ Films on $\mathrm{Si}<111>$ Substrate Grown by Low Energy $\mathrm{O}^{+}$Assisted Pulse Laser Deposited Technology," Materials Letters, Vol. 61, No. 14-15, 2006, pp. 29612964. doi:10.1016/j.matlet.2006.10.047

[12] K. S. Ratidar, D. Patidar, Y. Janu and N. S. Saxena, "Structural and Optical Charcterization of Chemically Synthesized ZnS Nanoparticles," Calcogenide Letters, Vol. 5, No. 6, 2008, pp. 105-110.

[13] G. P. Jushi, N. S. Saxena, T. P. Sharma, Y. Dixit and S. C. K. Mishra, "Bandgap Determination of Chemically Doped Polyaniline Materials from Reflectance Measurements,” Indian Journal of Pure \& Applied Physics, Vol. 41. No. 6, 2003, pp. 462-465.

[14] M. de Dios a, F. Barroso, C. Tojoa, M.C. Blanco and M. A. L'opez-Quintela, "Effects of the Reaction Rate on the Size Control of Nanoparticles Synthesized in Microemulsions," Colloids and Surfaces A: Physicochemical and Engineering Aspects, Vol. 270-271, 2005, pp. 83-87.

[15] X. Lu, T.Hanrath, K. P. Johnton and B. A. Kargel, "Growth of Single Crystal Silicon Nanowires in Supercritical Solution from Tethered Gold Particles on a Silicon Substrate,” Nano Letters, Vol. 3, No. 1, 2003, pp. 9399. doi:10.1021/nl0202307 